\title{
Past 30 days
}

National Cancer Institute

\section{Source}

National Cancer Institute. Past 30 days. NCI Thesaurus. Code C131330.

A period of time consisting of the 30 days before the present. 